\title{
Subject Index Vol. 53, 1994
}

\section{Adenocarcinoma 110}

-, renal 40

Angiography 166

Anticancer drugs 12

Antisperm antibody 143

Appendix 97

Arterial administration 117

Art erio venous fistula, postnephrectomy 99

Asymptomatic infertile men 217

Autologous tumor vaccine 6

Autosomal aberration 107

Azoospermia 222

Benign prostatic hyperplasia 196 Bladder 227

cancer cells 12

carcinoma 1

substitution with stomach, advantages 24

- $\quad$ urothelium 155Bone metastasis 172Brachytherapy 209Burch colposuspension

150Bypass, cardiopulmonary 38

Calcification 110 Cancer genetics 1 Carcinoma 125 -, prostate 135 Catheter toxicity 155 Cell

cycle 12

- viability 12 Chemoimmunotherapy 6Children 162

Chlamydiatrachomatis Ill Chordee without hypospadia 107 Crista urethralis 34

Cyclophosphamide 6

Cyst 162 Cystoscopy 186

Desmoid tumor 48

Detubularized intestinal substitute 30

Dialysis 79

Digital rectal examination 186

DNA content 181

- ploidy 18

Ejaculation 34 Ejaculatory duct 139 Embolization, selective 99 Enzymes 12

Epidermal cyst 53

- $\quad$ growth factor receptor 18Epididymis 222

Erectile dysfunction 214

Extent of disease 130

Extracorporeal Shockwave lithotripsy 82

- $\quad$ - - , in situ 87

Female incontinence 150 Flow cytometry 135

Gastropouch, operative techniques and 
results 24 Germ cell tumor 176 Gunshot injuries 92

Hemospermia 139 High energy shock wave 12 Histopathology 79 Horseshoe kidney 158

Hydronephrosis 102, 158

Immunohistochemistry 135

Incidental carcinoma, prostate 196

Incontinence pads 68

Infertility 222

Interferon- $\alpha 172$

Interleukin-2 6

Intracardiac tumor thrombus 38

Ki-67 index 18 Kidney 158, 162

neoplasms 172

-, diagnosis 230

-, radionuclide imaging 230

Late relapse 176 Leiomyosarcoma 166 Leukaemia 225 Linsidomine chlorhydrate 214 Lymph 104 Lymphagioma 104 Lymphocytic infiltration 225 Lymphokine-activated killer cells 117 Magnetic resonance imaging 191

Malignant potential 135

Metaplasia 110

Metastases 50

Mumps 179

Muscarinic cholinergic receptor 62

Musculus ejaculatorius 34

Needle biopsy 186 Neoplasms, multiple primary 230 Nephroblastoma 38 Nephrogenic adenoma 227 Nitric oxide 214

Oestrogen, oral 74 Orchidectomy 74 Orchitis 179

p53gene 1

Papillomavirus, human 125 Penile block 147

injuries 92

secondaries 135 Pericentric inversion 107 Perineum 53 Peripheral circulation 74

Pheochromocytoma 44 PMNelastase 217 Polymerase chain reaction 125 Postoperative pain

control 147 Prognosis 1, 196 Prognostic factors 130 Proliferation rate (Ki-67) 181 Prostaglandin Ei 214 Prostate 68,139,225

cancer 209

carcinoma 1 Prostate-specific antigen 186 Prostatic cancer 130

carcinoma 50,74

neoplasms 191

Radiation therapy 48 Radical prostatectomy 68, 196 RB gene 1 ai-Receptor 62 c1/8-Receptor 62

ß-Receptor 62 Renal calculi 82

cell carcinoma 79, 117, 181

endothelin 57

vein 44

Segmental difference 62 Semen 217 Seminal emission 34

- $\quad$ vesicle 139 Seminoma 48

Serum agglutinin titres 143 Skin metastasis 114

Studer-Zingg neobladder 30 Superficial operation, penis 147 
Temporary interruption 44 Testicular neoplasms 222 Tomography, X-ray computed 230 Transitional cell carcinoma 18, 114 Transrectal ultrasonography 139, 186 Transurethral radiotherapy 209 Tumo(u)r progression 1 - suppressor genes 1

Ultrasonic diagnosis 191 Ultrasonography 230 Ultrasound 162 Urachus 110

Uracil and tegafur combination Ureaplasma urealyticum 217 Ureter substitution 97 Ureteral neoplasm 166

obstruction 102

polyp 102

stone 87 Ureteric obstruction 57 Urethra 104 Urethralcyst 169

injuries 92

tumors 104 Urethroplasty 92 Urinary bladder 114

cytology 18

incontinence 68

172 Urodynamic investigation 30

- studies 150 Urodynamics 68 Urological tumours 1

Vaccination 179 Vas deferens, dog 62 Vasectomy 143 Vasovasostomy 143

Weanling rats 57 Wilms tumor 38

2. 236

Subject Index 